\title{
Qualidade de vida de professores do ensino fundamental: uma perspectiva para a promoção da saúde do trabalhador
}

\author{
Quality of life elementary school teachers: a \\ perspective for health promotion of worker
}

Vera Maria da Rocha, ${ }^{1,2}$, Marcos Henrique Fernandes, ${ }^{2,3}$

\section{RESUMO}

A qualidade de vida é um importante aspecto a ser considerado na promoção de saúde dos professores, sobre os quais vêm sendo atribuídas diversas funções no cotidiano de suas atividades de trabalho. Objetivo: Avaliar a qualidade de vida dos professores do ensino fundamental do município de Jequié-BA. Método: A pesquisa foi realizada com uma amostra aleatória constituída por 91 professores que responderam o questionário genérico de avaliação da qualidade de vida (SF-36). O questionário analisa oito domínios relativos à qualidade de vida. Os dados foram analisados por meio da estatística descritiva, com determinação de médias, freqüências e desvio-padrão. Resultados: Todos os domínios do

\section{Palavras-chave}

Docente, ensino fundamental, qualidade de vida.

\section{Key-words}

Teacher, elementary education, quality of life. SF-36 apresentaram-se prejudicados com destaque para vitalidade $(46,26)$ e dor $(53)$, como os de menor escore, e capacidade funcional $(65,71)$ e limitação por aspectos emocionais (62,63), como os de maior escore. Conclusão: A qualidade de vida da população estudada encontra-se comprometida, o que pode repercutir no estado de saúde de tais indivíduos.

\begin{abstract}
Quality of life is an important aspect of teacher health, since many tasks have been assigned to them as part of their daily work routine. Objetive: To evaluate the quality of life of elementary school teachers in the city of Jequié, Brazil. Methods: The research was carried out with a random sample consisting of 91 teachers who had responded to the generic (SF-36) quality of life questionnaire, which analyzes eight domains related to quality of life. Descriptive statistics were used to analyze the data, which were expressed in means, frequency and standard deviation. Results: All the areas of the SF-36 were compromised. The lowest scores were obtained for vitality (46.26) and pain (53), whereas and functional capacity (65.71) and emotionally-related limitations (62.63) recorded the highest values. Conclusion: The quality of life of this population was low, which may be influencing the health status of these individuals.
\end{abstract}

1 Universidade Federal do Rio Grande do Sul (UFRGS). 2 Programa de Pós-Graduação em Ciências da Saúde da Universidade Federal do Rio Grande do Norte (UFRN). 3 Universidade Estadual do Sudoeste da Bahia (UESB).

Endereço para correspondência: Marcos Henrique Fernandes

Rua Carlos Andrade, 515, Jequiezinho - 45205-090 - Jequié, BA

E-mail: marcoshenriquefernandes@bol.com.br 


\section{INTRODUÇÃO}

O notável processo de globalização que a sociedade atual vive impõe um ritmo acelerado de produção tecnológica e altera de maneira profunda as relações desenvolvidas no mundo do trabalho, provocando, por conseqüência, queda crescente na qualidade de vida da população trabalhadora'. A escola se constitui um importante ambiente onde tal problemática está presente, sofrendo os impactos das mudanças políticas, tecnológicas e econômicas decorrentes da globalização.

Nesse contexto, o ambiente escolar não foge à regra capitalista, para a qual tem sido, cada vez mais, impostas novas demandas no processo de formação de crianças e jovens. Uma das categorias profissionais que mais tem exigido mudanças é a dos professores, em virtude da intensificação do ritmo de trabalho. Tradicionalmente, era atribuído a esses profissionais o papel referente ao ensino de disciplinas, tendo gradativamente assumido funções, como construir hábitos de saúde, assessoramento psicológico, educação para o trânsito, entre outras².

Somado à problemática citada anteriormente vem o excesso de tarefas burocráticas, a falta de autonomia e infraestrutura do ambiente escolar, as relações conflitantes com familiares de alunos e, principalmente, a baixa remuneração, tornando evidente o quadro crônico de depreciação e desqualificação social, psicológica e biológica dos professores. Emerge dessa situação um cenário com efeitos adversos, proporcionando aos docentes um conjunto de mal-estares, em muitos casos desestabilizando a economia psicossomática e gerando doenças diversas, que influenciam fortemente na qualidade de vida destes profissionais ${ }^{3}$.

Qualidade de vida foi definida, de modo mais genérico, como "a percepção do indivíduo de sua posição na vida no contexto da cultura e sistema de valores, nos quais ele vive em relação aos seus objetivos, expectativas, padrões e preocupações ${ }^{14}$. Dois aspectos são relevantes dentro do conceito de qualidade de vida: a subjetividade, que considera a percepção da pessoa sobre o seu estado de saúde e sobre os aspectos não médicos do seu contexto de vida; e a multidimensionalidade, que se refere ao reconhecimento de que o construto é composto por diferentes dimensões 5 .

$\mathrm{Na}$ concepção de trabalhar a saúde coletivamente no ambiente escolar, não se pode perder de vista a noção de saúde individual, o que remete à reflexão sobre a saúde dos professores com os quais se pretende trabalhar, pois uma escola promotora de saúde deve incluir a idéia do docente saudável, possuindo bem-estar em diversos aspectos, como físico, mental, espiritual, entre outros.

A proposta das escolas promotoras de saúde, lançadas pela Organização Pan-Americana da Saúde (OPAS/OMS) em 1995, reafirma a prática de promoção de saúde, proposta pela Carta de Ottawa, a qual consiste do "processo de capacitação da comunidade para atuar na melhora da sua qualidade de vida e saúde, incluindo maior participação no controle deste processo"6. O professor, portanto, deve ser capacitado a cuidar de si e agir em grupo na defesa da promoção de qualidade de vida, devendo perceber a escola como espaço de humanização e promoção de saúde, onde as práticas educativas não devam se limitar às tradicionais ações pedagógicas, e, sim, a possibilitar também as transformações individuais e sociais?.

Nessa perspectiva, existe pouco conhecimento sobre as condições de trabalho e a vida do professor na escola, principalmente sobre a qualidade de vida de docentes do ensino fundamental, os quais, geralmente, subestimam as suas necessidades de saúde e se conformam com o quadro desanimador em que se encontram, o que chama a atenção para a necessidade do desenvolvimento de ações de promoção de saúde para este grupo de trabalhadores. Assim, este estudo objetivou avaliar a qualidade de vida dos professores do ensino fundamental do município de Jequié-BA.

\section{METODOLOGIA}

Trata-se de um estudo descritivo de corte transversal. A amostra da pesquisa foi constituída por 91 professores do ensino fundamental da rede municipal de Jequié-BA, número obtido segundo o cálculo amostral com intervalo de confiança de $95 \%$.

Como instrumento de pesquisa, utilizou-se o Short Form (SF) 36 do instrumento Medical Outcomes Study (MOS), que é um questionário multidimensional composto por 36 itens, tendo sido traduzido e validado para a população brasilei$r a^{8}$. Este instrumento foi criado com a finalidade de avaliar a qualidade de vida relacionada à saúde de maneira genérica, não possuindo conceitos específicos para determinada idade, doença ou grupo de tratamento. O SF-36 é formado por oito componentes: capacidade funcional (dez itens), aspectos físicos (quatro itens), dor (dois itens), estado geral de saúde (cinco itens), vitalidade (quatro itens), aspectos sociais (dois itens), aspectos emocionais (três itens), saúde mental (cinco itens) e mais uma questão de avaliação comparativa entre as condições da saúde atual e a de um ano antes.

Para avaliar os resultados, foi dado um escore para cada questão, objetivando transformá-lo numa escala graduada de 0 a 100, porquanto o zero (0) correspondia a um pior estado de saúde e cem (100) a um melhor estado, possibilitando a análise individual de cada dimensão. Não existe um único valor que resuma toda a avaliação, para que não se caia no erro de não identificar os verdadeiros problemas relacionados à saúde do indivíduo investigado. Utilizou-se a 
estatística descritiva com determinação de médias, freqüência e desvio-padrão, para análise dos dados.

O projeto de pesquisa foi aprovado pelo Comitê de Ética em Pesquisa da Universidade Federal do Rio Grande do Norte (UFRN), incluindo a assinatura do termo de consentimento livre e esclarecido.

\section{RESULTADOS}

Dos 91 docentes que constituíram a amostra deste estudo, 87 (95,60\%) eram do sexo feminino e 4 (4,40\%) do sexo masculino, com média de idade de 36,05 anos, possuindo desvio-padrão (DP) de 6,98. Em relação ao estado civil, 62 $(68,13 \%)$ casados, a maioria, seguidos de $14(15,38 \%)$ solteiros, 12 (13,18\%) desquitados, 2 (2,2\%) viúvos e 1 (1,1\%) não respondeu tal questionamento. Dos professores avaliados, 82 (90,10\%) possuem regime de trabalho de 40 horas semanais, 6 (6,50\%) trabalham 20 horas semanais, 2 (2,19\%) têm regime de 50 horas semanais e 1 professor não respondeu tal questionamento.

Os resultados referentes à avaliação da qualidade de vida estão descritos na Tabela 1, que apresenta as médias de cada domínio do SF-36.

De modo geral, pode-se observar que os valores inferiores a 70 pontos encontrados em todos os domínios, que a qualidade de vida não pode ser avaliada de maneira positiva para a categoria de trabalhadores estudada. $O$ domínio que apresentou menor escore foi vitalidade $(46,26)$ e os com maiores escores foram capacidade funcional $(65,71)$ e limitação por aspectos emocionais $(62,63)$. Na Tabela 1 ainda estão descritos os valores mínimos, os valores máximos e os desvios-padrão para cada domínio.

Tabela 1. Distribuição dos professores segundo a qualidade de vida

\begin{tabular}{lcccc}
\hline \multirow{2}{*}{ Domínio } & \multicolumn{2}{c}{ Qualidade de vida } & & \\
\cline { 2 - 3 } \cline { 5 - 5 } & Média & Desvio-padrão & & Variaçäo (AV) \\
\hline CF $^{1}$ & 65,71 & 22,72 & & $5-100$ \\
LAF $^{2}$ & 61,53 & 33,62 & & $0-100$ \\
Dor & 53,00 & 19,77 & & $12-100$ \\
$\mathrm{EGS}^{3}$ & 62,62 & 17,58 & & $10-95$ \\
Vitalidade & 46,26 & 19,15 & & $5-90$ \\
$\mathrm{AS}^{4}$ & 62,08 & 23,25 & & $12,5-100$ \\
$\mathrm{LAE}^{5}$ & 62,63 & 38,85 & & $0-100$ \\
$\mathrm{SM}^{6}$ & 60,30 & 17,29 & & $16-92$ \\
\hline
\end{tabular}

Fonte: Dados coletados pelos autores.

'Capacidade funcional; ${ }^{2}$ Limitação por aspectos físicos; ${ }^{3}$ Estado geral de saúde; ${ }^{4}$ Aspectos sociais; ${ }^{5}$ Limitação por aspectos emocionais; ${ }^{6}$ Saúde mental.

\section{DISCUSSÃO}

O fato de 95\% dos docentes investigados no presente estudo serem do sexo feminino e do comprometimento de todos os domínios de qualidade de vida chama a atenção para a possível interferência de tal associação nos resultados anteriormente citados. O expressivo percentual de mulheres como professoras do ensino fundamental é praticamente uma constante em estudos com esta população, episódio que é decorrente do processo histórico de expansão do setor educacional ocorrido no Brasil e da entrada das mulheres no mercado de trabalho, no qual essas foram chamadas a exercer o papel de mães educadoras ${ }^{9}$.

Acrescentando à problemática citada anteriormente, vem o fato de que essas trabalhadoras exercem também outra jornada de atividades em seus domicílios, decorrentes das obrigações socialmente atribuídas às mulheres, como cuidado com a casa e filhos, tarefas estas que, quando somadas com às desenvolvidas no ambiente escolar, podem gerar diversos problemas de saúde decorrentes da sobrecarga.

Alguns estudos foram realizados com professores sobre qualidade de vida, visto que esses investigaram populações de docentes universitários, os quais possuem regime de trabalho um pouco diferenciado daqueles que trabalham com o ensino fundamental ${ }^{10,11}$.

Foi encontrado na literatura apenas um estudo que avaliou a qualidade de vida de professores do ensino fundamental, no qual foram investigados 142 professores da rede pública de ensino de uma cidade do interior do Estado de São Paulo, apresentando como critério de inclusão a presença de sintomatologia dolorosa de origem osteomuscular. Entre os principais resultados descritos na pesquisa citada, os domínios dor $(59,60)$ e da vitalidade $(59,80)$ foram os mais prejudicados, seguidos de aspectos sociais $(68,90)$, saúde mental $(68,40)$ e limitação por aspectos emocionais $(66,60)^{12}$. Apesar de o estudo realizado com os professores da cidade de Jequié-BA não ter se limitado a investigar docentes com queixas dolorosas, os mesmos domínios também apresentaram escores baixos, sendo a vitalidade o domínio mais comprometido em ambos os estudos.

Três domínios entre os professores de São Paulo apresentaram valores acima da média de 70 pontos, são eles: capacidade funcional $(79,30)$, aspectos físicos $(76,0)$ e estado geral de saúde $(75,60)$. Nenhum domínio conseguiu atingir o escore de 70 pontos entre os docentes investigados em Jequié-BA, demonstrando importante comprometimento da qualidade de vida de tais trabalhadores, sendo a capacidade funcional o domínio que mais se aproximou desta pontuação com o escore de 65,71.

Em relação específica aos resultados encontrados em cada domínio, a capacidade funcional está relacionada à aptidão física, ou seja, à capacidade de realização de atividades diárias de um indivíduo, que tem de resistir e superar desafios e o estresse ${ }^{13}$. A avaliação deste realizada pelo SF-36 não se deteve à distinção específica das atividades, tratando apenas de avaliar, de maneira genérica, a influência que 
a capacidade funcional e a vitalidade exerciam sobre a qualidade de vida do docente. O domínio "capacidade funcional" possuiu média de 65,71 e "vitalidade" obteve média de 46,26, o que leva à interpretação de que o grupo analisado possuía regular capacidade funcional e vitalidade ruim.

Foi identificado, em um estudo realizado com 250 professores do ensino fundamental em Vitória da ConquistaBA, que vários aspectos contribuem para a diminuição da vitalidade do professor. Entre esses, os mais fortemente percebidos foram: ritmo acelerado de trabalho $(67,9 \%)$, ritmo frenético de trabalho $(54,9 \%)$, posições da cabeça e braços inadequadas e incômodas $(53,4 \%$ ) e longos períodos de intensa concentração em uma mesma tarefa $(51,9 \%)^{9}$.

Outro estudo sobre as condições de saúde de professores foi o desenvolvido no município de Vitória-ES, onde foram investigados 607 docentes da rede pública de ensino, sendo verificado também que as principais fontes de tensão e cansaço foram: a má remuneração pelo exercício da profissão, o ritmo intenso de trabalho e o número excessivo de alunos em sala de aula ${ }^{14}$.

Levando-se em consideração que os entrevistados do presente estudo tiveram, em conjunto, baixo valor para o domínio "vitalidade", aspectos negativos como os anteriormente citados podem ser os determinantes para tal resultado.

Todo o trabalho é gerador de fatores desgastantes e potencializadores, que são determinantes dos processos saúde-doença vivenciados pelos trabalhadores e da qualidade de vida no trabalho' ${ }^{15}$.

Observou-se, no presente estudo, que os indivíduos possuem regular escore para aspectos físicos (média de 61,53), chamando a atenção para o fato de que a saúde física prejudicada pode incidir na prática da docência, acarretando maior número de faltas ao trabalho, assim como maior desinteresse na realização e inovação da prática educacional.

Em alguns estudos mais recentes que buscaram compreender as condições de trabalho e a saúde de professores $^{13,16}$, foi observado que aspectos da docência, como ficar em pé por várias horas, eram fatores que os limitavam fisicamente. As limitações físicas reveladas nesses estudos envolviam basicamente queixas de má postura corporal e queixas relacionadas à voz.

No que se refere ao domínio "dor" do questionário aplicado aos docentes, obteve-se a média de 53 pontos, representando o segundo item mais prejudicado. No estudo realizado sobre qualidade de vida com professores do ensino fundamental em uma cidade do estado de São Paulo, a dor foi o domínio mais comprometido com escore de 59,6, valor próximo do encontrado com docentes de Jequié-BA' ${ }^{12}$. A prática da docência, em longo prazo, pode levar a várias patologias musculoesqueléticas e psicológicas, muitas vezes relacionadas a quadros álgicos intensos, podendo acarretar absenteísmos ${ }^{16,17}$. A docência vem sofrendo constantes alterações no decorrer de sua história, embora o professor não tenha tido condições de criar meios para se adaptar às mudanças, levando ao surgimento de desequilíbrios na estrutura corporal e em dor $r^{17}$.

No domínio relacionado ao estado geral de saúde dos docentes investigados, obteve-se o valor médio de 62,62, o que indica má avaliação da qualidade de vida deste item na população investigada, podendo este resultado ter repercussão direta na prevalência de morbidades entre tais indivíduos, refletindo em afastamento da profissão da docência.

A saúde dos trabalhadores é conseqüência da relação complexa e dinâmica entre o trabalhador e a sua atividade de trabalho. O dinamismo no processo de trabalho e a velocidade nas mudanças tecnológicas e organizacionais extrapolam o limite e o reducionismo das visões estáticas e monocausais na relação trabalho e saúde. A noção de carga de trabalho tenta superar isso, pressupondo a análise do processo de trabalho de maneira dinâmica e abrangente, incluindo também a organização do trabalho $0^{3,15}$.

Os problemas de saúde dos docentes têm sido estudados a partir dos anos 1960, na Europa e no Brasil, a partir da década de 1970. Na década de 1980, vários autores centraram suas reflexões sobre a educação chamando a atenção para a figura do docente e buscaram decifrar os efeitos que podiam estar sofrendo pela racionalização de seus trabalhos ${ }^{18}$.

No estudo realizado com os professores da cidade de Vitória da Conquista-BA, ficou demonstrado que o percentual de docentes com diagnósticos médicos de saúde, desde que começaram a trabalhar, foi bastante elevado, visto que os mais freqüentes foram varizes em membros inferiores e lesões por esforços repetitivos, doenças que são potencialmente relacionadas ao trabalho. Um terço destes professores referiu problemas de saúde nos 15 dias anteriores à entrevista, o que pode ser traduzido num elevado absentismo ao trabalho?.

Outro estudo realizado em Málaga, utilizando os arquivos de inspeção médica da delegação de Educação e Ciência com 13.183 professores, revelou que as causas relativas à saúde mais importantes de licença de professores foram traumatológicas, geniturinárias, obstétricas e neuropsiquiátricas. Alguns desses professores, considerados pela Inspeção médica como doentes mentais crônicos, são destinados, ao final de cada ano, a tarefas administrativas, afastando-se, assim, das aulas ${ }^{17}$.

Em relação ao domínio "aspectos sociais", que envolve a interação familiar, com amigos e com a comunidade, obteve-se o valor médio para os docentes de Jequié-BA de 62,08 , indicando que possuem regular interação social.

Já os resultados dos domínios limitação por "aspectos emocionais" $(62,63)$ e "saúde mental" $(60,30)$ revelam que os professores investigados no presente estudo possuem regular saúde mental, indicando que os fatores estressan- 
tes do trabalho devem estar influenciando e determinando a saúde mental destes docentes, o que pode levá-los a adquirir a síndrome de Burnout. No estudo sobre qualidade de vida também realizado com professores do ensino fundamental, o valor encontrado para o domínio "aspectos emocionais" foi 66,6 , próximo também ao encontrado para a população na presente pesquisa ${ }^{12}$.

Várias pesquisas apontam distúrbios psíquicos associados às características do conteúdo do trabalho na classe de professores. A prevalência dos distúrbios psíquicos nos estudos observados foi bastante variada, situada na faixa de $8,7 \%$ a $41,5 \%{ }^{13-16}$.

As características mais estressantes do trabalho docente são: trabalho repetitivo, intensa concentração em uma mesma tarefa por um longo período, volume excessivo de trabalho, ritmo acelerado, interrupção das tarefas antes de serem concluídas, tempo insuficiente para realização das tarefas, falta de interesse dos colegas de trabalho, exposição a hostilidades, conflitos com os colegas de trabalho e inexistência de processo democrático?.

No exercício profissional da atividade docente estão presentes diversos estressores psicossociais, alguns relacionados à natureza de suas funções, outros relacionados ao contexto institucional e social onde estas são exercidas. Esses estressores persistentes podem levar à síndrome de Burnout, que tem vínculo com situações de trabalho, resultante da constante e repetitiva pressão emocional associada com intenso envolvimento com pessoas por longos períodos. Essa síndrome em professores afeta o ambiente educacional e interfere na obtenção dos objetivos pedagógicos, ocasionando problemas de saúde e absenteísmo e intenção de abandonar a profissão' 19 .

\section{CONCLUSÕES}

De forma geral, foi observado que todos os domínios investigados pelo SF-36 com os professores do ensino fundamental do município de Jequié-BA encontram-se prejudicados, destacando-se a vitalidade e a dor, as quais apresentaram menor escore. Este resultado chama a atenção para a necessidade da realização de intervenções com tal população, tanto em nível de políticas públicas que possibilitem o desenvolvimento de um trabalho docente adequado, que objetive a promoção de saúde destes trabalhadores, quanto em nível de ações de profissionais de saúde que possam minimizar os danos à saúde, advindos de tal prática profissional. Na perspectiva de criação de escolas promotoras de saúde, não se pode perder de vista a saúde dos professores como elemento indispensável na concretização desta proposta.
É de fundamental importância que sejam criadas discussões sobre educação e promoção de saúde no ambiente escolar, devendo evoluir para a operacionalização de programas que possibilitem o aumento da qualidade de vida dos professores.

\section{REFERÊNCIAS}

1. Heckert AL, Aragão E, Barros MEB, Oliveira S. A dimensão coletiva da saúde: uma análise das articulações entre gestão administrativa-saúde dos docentes, a experiência de Vitória. In: Athayde M, Barros MEB, Brito JC, Neves MY (Org.). Trabalhar na escola? Só inventando 0 prazer. Rio de Janeiro: IPUB/CUCA; 2001. p. 123-62.

2. Santomé JT. 0 professor em época de neoliberalismo: dimensões sociopolíticos de seu trabalho. In: Linhares C (Org.). Os professores e reinvenção da escola: Brasil e Espanha. São Paulo: Cortez; 2001

3. Gomes L. Trabalho multifacetado de professores/as: a saúde entre limites [dissertação]. Rio de Janeiro: Fundação Oswaldo Cruz; 2002.

4. The WHOQOL Group. The Word Health Organization quality of life assessment (WHOQOL): position paper from the World Health Organization. Soc Sci Med. 1995;41:1403-10.

5. Seidi EMF, Zannon CMLC. Qualidade de vida e saúde: aspectos conceituais e metodológicos. Cad Saúde Pública. 2004;20:580-8

6. WHO. Carta de Ottawa. In: Ministério da Saúde/FIOCRUZ. Promoção da Saúde: Cartas de Ottawa, Adelaide, Sundsvall e Santa Fé de Bogotá. Brasília, 1998. p. 11-8.

7. Moura JBVS, Lourinho LA, Valdês MTM, Frota MA, Catrib AMF. Perspectivas da epistemologia histórica e a escola promotora de saúde. História, Ciências, Saúde. Manguinhos; 2007:14:2:489-501

8. Ciconelli RM, Ferraz MB, Quaresma MR, Meinão I, Santos W. Tradução para língua portuguesa e validação do questionário genérico de avaliação de qualidade de vida SF-36 (Brasil SF-36). Rev Bras Reumato. 1999;39:3:143-50.

9. Delcor NS, Araújo TM, Reis EJFB, Porto LA, Carvalho FM, Silva MO, et al. Condições de trabalho e saúde dos professores da rede particular de ensino de Vitória da Conquista, Bahia, Brasil. Cad Saúde Pública. 2004;20:187-96.

10. Souza JC, Cardoso WL, Damasceno C, Souza N, Siqueira AF. Qualidade de vida de professores universitários. J Bras Psiquiatria 2004; 53: 263-266.

11. Almeida, LGD, Porto GG. Análise das condições de vida de professores universitários [monografia de conclusão de curso]. Jequié: Universidade Estadual do Sudoeste da Bahia.

12. Carvalho AJFP, Alexandre NMC. Qualidade de vida e sintomas osteomusculares relacionados ao trabalho em professores do ensino fundamental. Fisioterapia Brasil. 2006;7:279-84.

13. Martins M0. Estudo dos fatores determinantes da prática de atividades físicas de professores universitários [dissertação]. Santa Catarina (PR): Universidade Federal de Santa Catarina (UFSC), 2000.

14. Marchiori F, Barros MEB, Oliveira SP. Atividade de trabalho e saúde dos professores: 0 programa de formação como estratégia de intervenção nas escolas. Trabalho, Educação e Saúde. 2005:3:143-70.

15. Rocha SSL, Felli VEA. Qualidade de vida no trabalho docente em enfermagem. Rev LatinoAm Enfermagem. 2004;12:28-35.

16. Silvany Neto AM, Araújo TM, Dutra FRD, Azi GR, Alves RL, Kavalkievicz C, et al. Condições de trabalho e saúde de professores da rede particular de ensino de Salvador, Bahia. Rev Baiana de Saúde Pública. 2002;24:42-56.

17. Esteve JM. 0 mal-estar docente: a sala de aula e a saúde dos professores. Bauru, SP: Ed. Universidade do Sagrado Coração; 1999.

18. Martinez MC, Paraguay AIB, Latorre MRDO. Relação entre satisfação com aspectos psicossociais e saúde dos trabalhadores. Rev Saúde Pública. 2004:38:55-61.

19. Carlotto MS. A síndrome de burnout e o trabalho docente. Psicol Estud. 2002;7:21-9. 